\title{
QUALITY INSPECTION AND ANALYSIS OF THREE-DIMENSIONAL GEOGRAPHIC INFORMATION MODEL BASED ON OBLIQUE PHOTOGRAMMETRY
}

\author{
Dong Shuai ${ }^{1, *}$, Yan Qingqing ${ }^{1}$, Xu Yongmin ${ }^{1},{\text { Bai } \operatorname{Jin}^{1}}^{1}$ \\ ${ }^{1}$ National Quality Inspection and Testing Center for Surveying and Mapping Products, Beijing, China- dongshuai@ vip.qq.com
}

Commission VI, WG III/IVb

KEY WORDS: Oblique Photogrammetry, Three-Dimensional Geographic Information Model, Quality Inspection, Basic Principles, Technical Features, Common Problems.

\begin{abstract}
:
In order to promote the construction of digital geo-spatial framework in China and accelerate the construction of informatization mapping system, three-dimensional geographic information model emerged. The three-dimensional geographic information model based on oblique photogrammetry technology has higher accuracy, shorter period and lower cost than traditional methods, and can more directly reflect the elevation, position and appearance of the features. At this stage, the technology of producing threedimensional geographic information models based on oblique photogrammetry technology is rapidly developing. The market demand and model results have been emerged in a large amount, and the related quality inspection needs are also getting larger and larger. Through the study of relevant literature, it is found that there are a lot of researches on the basic principles and technical characteristics of this technology, and relatively few studies on quality inspection and analysis. On the basis of summarizing the basic principle and technical characteristics of oblique photogrammetry technology, this paper introduces the inspection contents and inspection methods of three-dimensional geographic information model based on oblique photogrammetry technology. Combined with the actual inspection work, this paper summarizes the quality problems of three-dimensional geographic information model based on oblique photogrammetry technology, analyzes the causes of the problems and puts forward the quality control measures. It provides technical guidance for the quality inspection of three-dimensional geographic information model data products based on oblique photogrammetry technology in China and provides technical support for the vigorous development of three-dimensional geographic information model based on oblique photogrammetry technology.
\end{abstract}

\section{INTRODUCTION}

The digital city concept based on multi-source spatial information is a key node in the digital earth construction process, and is an inevitable trend of urban development and social informatization. It realizes deep integration of $3 \mathrm{~S}$ technologies (geographical information systems, remote sensing systems, and global positioning systems), and the internet and virtual technology. As an important part of a digital city, the surveying accuracy and visibility of city three-dimensional model is directly related to the construction efficiency, scientific decision-making, delicacy management, and integration and sharing of information resources of the digital city.

The traditional photogrammetry technology mainly obtains the top information features of the city buildings using aviation and satellite remote sensing, and then rebuilds the model. However, the traditional photogrammetry technology has very limited access to outline information and texture information on the side of the object. With the development of aerial photography and the urgent need for more realistic, more complete and highprecision urban $3 \mathrm{~d}$ models, oblique photogrammetry has emerged. Urban three-dimensional geographic information model based on tilted photogrammetry technology not only can significantly improve the model production efficiency, but also can more intuitively reflect the position geometry, surface texture and its attributes and other information of the relevant geographical elements in the three-dimensional space, to make up for the deficiency of the orthophoto map.
Many domestic experts and scholars have carried out a large amount of basic research work on oblique photogrammetry technology. Li Ying et.al proposed an automated real-world 3D model production process using Low-altitude camera technology (Li Ying et.al, 2017a). Yang Xinli et.al reviewed the main process and technological features of oblique photography technology and provided a good The theory reference for the production of 3D modelling technology (Yang Xinli et.al, 2017a). Liu Yang et.al analyzed the data processing process and key technologies of UAV oblique photogrammetry technology, discovered the necessity of the distortion correction in UAV capture images, and established the three-dimensional model using Smart3D Capture system (Liu Yang et.al, 2016).Tan Jinshi et.al introduced the entire production process based on the three-dimensional modelling of Qingyuan city in Guangdong province and a county in Shanxi Province, and evaluated the plane and elevation accuracy of the model to prove that the oblique photogrammetry technology can accurately reflect the features of landforms under the national surveying and mapping accuracy requirements, and are suitable for the construction of urban three-dimensional models in largescale areas (Tan Jinshi et.al, 2015a and Zhou Jie, 2016a). Chen Xingfang et.al introduced the data production process and its application fields based on data production of the real threedimensional model of Xining City in the plateau region (Chen Xingfang et.al, 2016a).

In summary, many experts and scholars mainly introduce the basic principles of the production of three-dimensional models based on oblique photogrammetry technology using the establishment of a urban three-dimensional model, but it does

*Corresponding author: Dong Shuai (1992-), Assistant engineer, E-mail:dongshuai@vip.qq.com. 
not involve the specifications for inspection and acceptance of quality of three-dimensional model data products on geographic information. The three-dimensional model data on geographic information shall be modelled, produced, managed and applied according to the "Specifications for the Digital Products of Three-Dimensional Model on Geographic Information" (National Administration of Surveying, Mapping and Geoinformation, CH/T 9015-2012) , and the related data product quality and accuracy level shall be detected and evaluated based on "Specifications for Inspection and Acceptance of Quality of Three-Dimensional Model Data Products on Geographic Information " (National Administration of Surveying, Mapping and Geoinformation, CH/T 9024-2014). Therefore, on the basis of summarizing the basic principles and technical characteristics of oblique photogrammetry technology, this paper introduces the inspection contents and inspection methods of three-dimensional model on geographic information based on oblique photogrammetry technology. It combines the actual inspection work, summarizes the quality problems of three-dimensional model on geographic information based on oblique photogrammetry technology, analyzes the causes of the problems, and put forwards quality control measures. It also provides technical support for the vigorous development of three-dimensional model on geographic information based on oblique photogrammetry technology.

\section{PRODUCTION OF THREE-DIMENSIONAL MODELS BASED ON OBLIQUE PHOTOGRAMMETRY}

\subsection{Basic Principles of Oblique Photogrammetry}

Oblique photogrammetry technology is a high technology developed in the field of international surveying and mapping in recent years.It has changed the limitations of previous aerial photogrammetry that can only use a single camera to photograph objects from a vertical angle, by carrying multiple sensors on the same flight platform. At the same time, images are collected from different angles such as vertical, side, front and rear views (Figure 1) to obtain more complete and accurate information on ground objects(Li Zhenzhou et.al, 2012a).

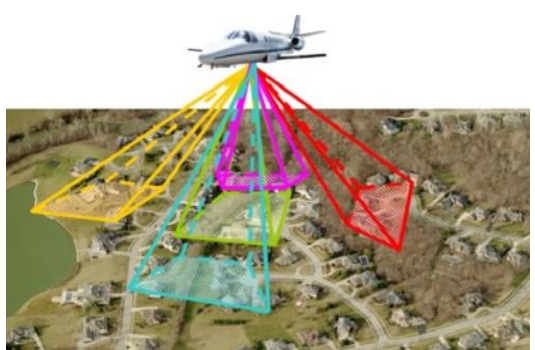

Figure 1. Multi-angle Aerial Photography

Using information such as flight altitude, exposure time, and latitude and longitude coordinates when shooting an image can greatly facilitate the analysis and processing of oblique images. The acquired multi-angle oblique image not only meets the needs of photogrammetric data processing, but also satisfies the requirement of $3 \mathrm{D}$ modelling for adding texture information. Through the process of combined adjustment, image matching and image rectification processing for oblique photographic measurement images, an image-based high-density point cloud model can be generated, and a three-dimensional model of oblique photographic measurement based on image texture can be generated.

\subsection{Technical Features and Benefits}

Compared with traditional orthophotos, oblique photogrammetry technology has the following technical features and advantages: (1) In terms of authenticity, the oblique images allows the user to view the feature from multiple angles, restore the characteristic of the feature to the greatest extent, and reflect the actual situation of the feature more realistically, which greatly compensates for the insufficiency of the orthographic image. (2) In the aspect of automation, the process of geometric correction and combined adjustment of multi-view images can be performed through specialized automated modelling software. The high-density point cloud based on oblique images is generated by operation, and the TIN model is constructed by point clouds to generate oblique photography $3 \mathrm{D}$ model. (3) In terms of technical application, single-image measurement can be achieved through the use of supporting software, and measurement including height, length, area, angle, and slope can be directly performed based on the result image, extending the application of the oblique photography in the industry. (4) In terms of economy, the use of the large-scale mapping of aerial photography for a variety of three-dimensional digital city applications, combined with the method of batch extraction and texture-matching from oblique images, not only achieves the collection of side textures of buildings, but also an effectively reduce the city's three-dimensional modelling costs. (5) In terms of resource sharing, compared to the application of threedimensional GIS technology for large-scale three-dimensional data, the amount of data acquired by the application of oblique photography technology is much smaller. The image data format can be quickly published on the web using mature technologies to achieve shared applications.

\section{THREE-DIMENSIONAL MODEL QUALITY EVALUATION BASED ON OBLIQUE PHOTOGRAMMETRY}

\subsection{Quality Inspection Work Overview}

In order to promote the construction of digital China's geospatial framework and speed up the construction of an information-based surveying and mapping system, the National Administration of Surveying, Mapping and Geoinformation initiated the construction of a digital city geospatial framework. Entrusted by some production unit, the National Quality Inspection and Testing Center for Surveying and Mapping Products carries out quality inspections of the three-dimensional geographic information models according to relevant technical specifications.

According to the relevant provisions of the "Specifications for Inspection and Acceptance of Quality of Three-Dimensional Model Data Products on Geographic Information" (National Administration of Surveying, Mapping and Geoinformation, CH/T 9024-2014), the testing contents include: spatial reference system, position accuracy, precision of expression, logical consistency, attribute accuracy, time accuracy, scene effects and attachment quality. However, the weights of different quality elements are different. According to their weights, the main quality elements (ordered by weight) are precision of expression (0.30), scene effects (0.20), and position accuracy (0.10).Among them, the precision of expression is mainly used to detect sub-elements such as terrain accuracy, DOM accuracy, TDOM accuracy, model fineness, and texture fineness. The scene effect mainly detects the scene integrity and coordination, and the positional accuracy is mainly used to detect sub- 
elements such as plane position accuracy, height accuracy, relative position between models, relative position of the model itself, etc. Through the detection of relevant quality subelements, the number of quality problems is determined and the product quality is finally assessed.

The detection methods mainly include: (1) Human-computer interaction inspection on indoor work. It mainly refers to the use of three-dimensional platform to detect the model. Under the specific software operating system environment, the data to be tested is visualized, and the data file is used as a carrier, through the form of graphics and images. Using computers to manually determine the correctness of the precision of expression, the completeness and coordination of the scene and other expression effects, the correctness of each attribute, logic compliance, file integrity, etc. (2) Field measurements on outdoor work. The sampling inspection method was used to check the integrity of the model, the accuracy of texture fineness, and the conformity and current situation of the scene effects. For position accuracy, a total station is usually used to detect the planar position accuracy and height accuracy. The relative position accuracy between the models is detected by using a handheld photoelectric distance meter or a RTK receiver to compare whether the position accuracy and its true value in the model meet the requirements of the relevant standards.

\subsection{Summary of the Quality of the Inspected 3D Model}

According to the relevant provisions in the " Specifications for Inspection and Acceptance of Quality of Three-Dimensional Model Data Products on Geographic Information" (National Administration of Surveying, Mapping and Geoinformation, $\mathrm{CH} / \mathrm{T}$ 9024-2014), through the inspection of three threedimensional model units in Xicheng District of Beijing, Xining City of Qinghai Province and Ya'an City of Sichuan Province, and the quality evaluation of the three-dimensional geographic information model, the overall quality of the results was found to be good. It shows that China has reached a relatively mature level in the field of three-dimensional geographic information model construction and can basically meet the general needs. However, the finesse of the model and the effect of the scene still need to be improved, leaving the research space for future generations to be still very broad.

\subsection{Common Problems of 3D Model Quality}

In the actual quality inspection and evaluation process, the following commonalities exist in the quality of the 3D model:

(1)There are loopholes and distortions in the model. For example, there are "breaking holes" in some housing building model (Figure 2), and some submarine models are deformed. Due to the lack of images caused by buildings obstructing each other and limited shooting angles, it is necessary to further adjust the shooting mode of the tilt camera or perform manual post-supplementation of the photos, and improve the relevant algorithms to self-check the "breaking holes" phenomenon.

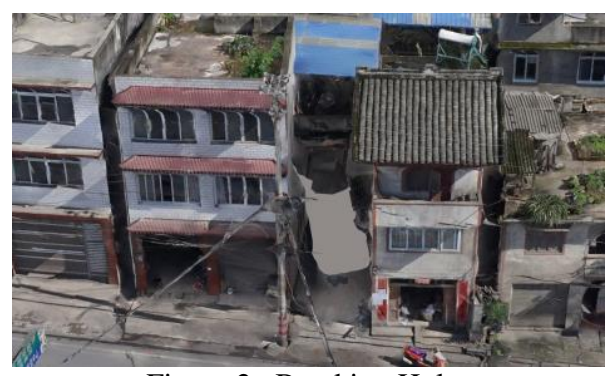

Figure 2. Breaking Holes

(2) The site model is distorted. For example, playgrounds and other open areas appear undulating phenomenon, mainly due to the single image, high similarity, local colour unevenness caused by computer identification difficulties and data filtering is not sufficient, this situation can be repaired by manual operation later.

(3) The model is incomplete (Figure 3). For example, trees, towers and other features have only the top model but no bottom model. This situation is mainly caused by the two following reasons: 1)The cover of the top floor causes the inability to obtain images of the underlying objects; 2)The structure of the ground object is relatively detailed, that makes the computer recognition difficult and the model establishment incomplete, so the supplement of photos is recommended.

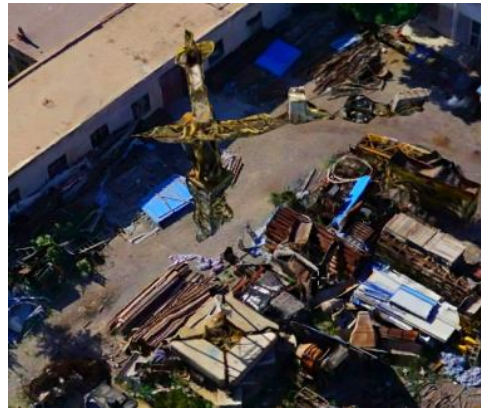

Figure 3. Incomplete Model

\section{CONCLUSION}

From the basic principles of oblique photogrammetry, this paper summarizes the technical features and advantages of the oblique photogrammetry technology, summarizes the detection content and detection methods of the three-dimensional model quality elements it has constructed, and indicates common problems of the three-dimensional model in the quality inspection and evaluation process. This article points out the research direction for the standardized production of threedimensional geographic information models for digital cities in China.

\section{ACKNOWLEDGEMENTS}

This study is sponsored by National Natural Science Foundation of China (41671440).

\section{REFERENCES}

Li Ying, Lin Zongjian, Su Guozhong, 2017a. Key Technology of Production of City Real 3D Images. Remote Sensing Information, 32(01), pp.35-39. 
Yang Xinli, 2017a. Study on 3D Digital City Modeling on the Basis of Oblique Photography Measurement Technology. Intelligent City, (02):5.

Liu Yang, 2016. Unmanned Aerial Vehicle (UAV) Oblique Photogrammetric Image Processing and 3D Modeling. East China University of Technology.

Tan Jinshi, Huang Zhengzhong, 2015a. Three Dimensional True Modeling and Precision Evaluation Base on Oblique Photogrammetry Technology. Modern Surveying and Mapping, 38(05), pp.21-24.

Zhou Jie, 2016a. Research on the Method of Constructing Realscence 3D Model Based on Oblique Photogrammetry Technology. Value Engineering, 35(25), pp.232-236.

Chen Xingfang, Ma Mingxia, Zhang Haihuan, Chang Hui, Liu Yuhang, 2016a. Research on 3D City Modeling of High Altitude Area Based on the Technique of Oblique
Photogrammetry. Geomatics \& Spatial Information Technology, 39(08), pp.149-152.

National Administration of Surveying, Mapping and Geoinformation. CH/T9015-2012, Specifications for the Digital Products of Three-Dimensional Model on Geographic Information. Surveying and Mapping Press, 2012.

National Administration of Surveying, Mapping and Geoinformation. CH/T 9024-2014, Specifications for Inspection and Acceptance of Quality of Three-Dimensional Model Data Products on Geographic Information. Surveying and Mapping Press, 2014.

Li Zhenzhou, Zhang Xuezhi, 2012a. Research on the Quick Construction of 3D Model of City Based on Oblique Photogrammetric Technique. Geomatics \& Spatial Information Technology, 35(04), pp.117-119. 